\title{
Multilinguales
}

1 | 2013

Pratiques littéraires, linguistiques, pédagogiques,

didactiques et médiations culturelles contemporaines

\section{Noms propres dans l'œuvre de Mohya : Entre tradition et innovation}

Proper nouns in Mohya's work: between tradition and innovation

\section{Mustapha tidjet}

\section{(2) OpenEdition}

Journals

Édition électronique

URL : https://journals.openedition.org/multilinguales/3348

DOI : $10.4000 /$ multilinguales.3348

ISSN : 2335-1853

Éditeur

Université Abderrahmane Mira - Bejaia

Édition imprimée

Date de publication : 1 juin 2013

Pagination : 159-166

ISSN : 2335-1535

Référence électronique

Mustapha tidjet, « Noms propres dans l'œuvre de Mohya : Entre tradition et innovation », Multilinguales [En ligne], 1 | 2013, mis en ligne le 01 juin 2013, consulté le 01 juillet 2021. URL : http:// journals.openedition.org/multilinguales/3348; DOI : https://doi.org/10.4000/multilinguales.3348

Ce document a été généré automatiquement le 1 juillet 2021.

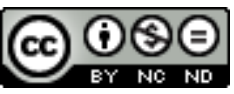

Multilinguales est mise à disposition selon les termes de la Licence Creative Commons Attribution Pas d'Utilisation Commerciale - Pas de Modification 4.0 International 


\title{
Noms propres dans l'œuvre de Mohya : Entre tradition et innovation
}

Proper nouns in Mohya's work: between tradition and innovation

\author{
Mustapha tidjet
}

1 La signification du nom propre joue un rôle important, parfois essentiel, dans la transmission du message dans certains domaines de la littérature amazighe, aussi bien dans la littérature traditionnelle que dans les modes modernes d'expression littéraire. Les pièces de Mohya en sont un parfait exemple.

2 Parce que c'est un auteur peu médiatisé, nous nous autorisons une brève biographie. Abdellah Mohya, plus connu sous le pseudonyme Mohend Ou Yehia, est un poète et dramaturge kabyle. Originaire des At Ouacifs. Il est né en 1950 à Azazga, wilaya de Tizi Ouzou. Il a fait ses études secondaires au lycée Amirouche de Tizi Ouzou. Après avoir obtenu son bac en 1968, il s'inscrit à l'université d'Alger pour préparer une licence en Mathématiques, qu'il obtient en 1972. En 1973, il s'installe en France pour étudier après avoir réussi le concours d'accès à l'école d'ingénieurs en hydraulique.

3 Il a publié bon nombre de travaux, aussi bien des créations que des traductions et adaptations vers le Kabyle d'œuvres reconnues. Son œuvre est composée de poèmes, de nouvelles, de pièces de théâtre, d'écrits divers. Grâce à des chanteurs populaires kabyles (Takfarinas, Malika Domrane, Slimane Chabi, Ferhat Imazighen Imoula), qui ont repris certains de ses poèmes, la poésie de Mohya est sortie de l'anonymat. Mais pour le poète Mohya, ce ne sont là que « chansonnettes² " (Mohya, 1985).

4 Ainsi, par le biais d'une description comparative des noms de personnages dans le conte kabyle et dans quelques productions de Mohya, nous allons essayer de mettre au jour les points de continuité entre les deux types de productions littéraires et les points de rupture qui font l'originalité de l'œuvre de cet écrivain. 


\section{Les noms dans la littérature de la tradition orale berbère}

5 En général, les noms propres dans la littérature de la tradition orale berbère, et en particulier kabyle, ne sont pas empruntés à la réalité. Ils appartiennent à un registre propre à ce type de production : Mqidec, Ğehhha, Belcejjud,...

6 Quand il est fait recours à un prénom kabyle courant, il subit un traitement qui va le distinguer de sa forme initiale, comme Eica (Aïcha) qui devient Eica Myizden (Aïcha, fille aux cendres). Un nom commun peut être également utilisé comme désignateur, surtout quand il s'agit d'êtres mythologiques, comme Wayzen (ogre), ou Teryel ou Tteryel (l'ogresse) du conte merveilleux.

7 En raison des connotations associées à chacun de ces noms propres, on les retrouve, en discours, dans des usages descriptifs ou qualificatifs ; c'est-à-dire qu'ils peuvent repasser dans le registre des noms communs par le procédé de l'antonomase :

Une espèce de synecdoque, par laquelle on met un nom commun pour un nom propre, ou bien un nom propre pour un nom commun. Dans le premier cas, on veut faire entendre que la personne ou la chose dont on parle excelle sur toutes celles qui peuvent être comprises sous le nom commun, et dans le second cas, on fait entendre que celui dont on parle ressemble à ceux dont le nom propre est célèbre par quelque vice ou par quelque vertu. (Dumarsais C.C., cité par Vaxelaire, 2005 : 270)

8 Ainsi, pour signifier la beauté exceptionnelle d'une jeune fille, elle sera qualifiée de Lounğa, (fille de l'ogresse d'une beauté sans pareille); quelqu'un de rusé, qui joue de mauvais tours, sera surnommé Mqidec.

Certains auteurs distinguent le cas où la dénomination implique un changement de catégorie grammaticale, c'est-à-dire l'utilisation du nom propre comme nom commun ou inversement, du cas où il n'y a pas de changement catégoriel. Dans le premier cas, ils parlent d'antonomase, alors que dans le second, ils préfèrent le terme de métaphore :

La métaphore, conçue comme métaphore vive, est une figure qui, contrairement à l'antonomase, se réalise dans le discours, n'implique pas de changement de catégorie grammaticale (le nom propre reste un nom propre) et ne correspond pas à un sens prévisible. N'importe quelle propriété du contenu du nom propre peut servir de base à une métaphore. (Gary-Prieur, $2001: 81$ )

C'est dans cette dernière catégorie que l'on peut classer les différents surnoms empruntés aux contes, comme Mqidec que l'on fait suivre de manière quasi systématique de sa périphrase : bu lehmum, ur yeggan ur yettnuddum (Mqidec à problèmes qui ne dort jamais et qui n'a pas sommeil).

11 Du statut de surnom, qui passe de génération en génération, le surnom se transforme en patronyme. C'est ce que nous avons relevé dans le cadre de notre thèse (Tidjet, 2013) pour les patronymes formés sur Meqidec ${ }^{3}$, Lounğa ${ }^{4}{ }^{4}$.

Sur le plan de leur formation linguistique, il est à remarquer qu'à côté des noms à morphologie simple, qui paraissent ordinaires ${ }^{5}$, comme c'est le cas de Lounğa et des emprunts à la littérature arabe (Ğehha), il y a eu également recours aux formations expressives comme Belcejjud Mqidec. Cependant, nous n'avons trouvé aucune racine avérée d'où serait dérivé Beleejjud, à moins qu'il n'ait été formé par association phonique avec mejjud, " gale $»^{6}$; ce qui paraît très probable. Il serait à rattacher à ijjed 
(adj.) « faible », «maladif », « mal formé », « dégénéré » (Dallet, 1982 : 361). On ne peut qu'avancer des hypothèses à ce sujet.

13 C'est le même cas pour Mqidec. Est-il formé sur qeddec qui est " attesté en berbère rifain comme verbe pour signifier "jouer un tour à quelqu'un" » (Bezzazi, 1999: 87) ? Ou bien dérive-t-il de aqeddac » domestique, serviteur » (Dallet, 1982:648) ? Ce dernier lexème étant aussi attesté dans l'arabe dialectal avec un sens très proche : qeddac « domestique qui fait les menus services (va à l'eau, fait les commissions,...) » (Beaussier, 1956 : 782).

\section{Les noms dans l'œuvre de Mohya}

14 L'analyse des noms propres dans une œuvre peut indiquer l'intention de l'écrivain, comme le signale D. de Camilli à propos de l'œuvre de Pavese :

En désirant connaître les racines, les origines de cette œuvre, il faut une approche particulière, c'est-à-dire l'analyse des noms propres que Pavese a employés et qu'il a cherchés dans plusieurs milieux, soit en ville soit à la campagne, voire Turin et les

Langhe, soit dans les littératures anciennes. (1998:348)

Mohya appartient à ces auteurs qui mettent un soin particulier à choisir et à confectionner les noms de leurs personnages; d'où ses créations onomastiques dans presque toutes ses œuvres. Les types de ces dénominations sont divers. Nous allons nous contenter de l'étude de quelques-uns à travers les noms de certains personnages principaux dans quatre textes de l'auteur.

\section{Ccix Aḥecraruf g-Gillulen Umalu}

16 Le nom du personnage principal est un nom commun attesté dans le kabyle, mais il est enrichi d'indications si précises qu'il crée un effet de réel.

17 En effet, d'abord d'un point de vue ethno-géographique, il est composé en partie d'un toponyme, Illulen Umalu, désignant le territoire d'une tribu connue en Kabylie.

Ensuite, ce toponyme est précédé d'un lexème emprunté à l'arabe, Ccix (« cheikh »), qui signifie maître ${ }^{7}$. Généralement, il désigne l'imam de la mosquée du village ou le maître d'école coranique ${ }^{8}$. Donc, il est assimilé à une particule nobiliaire.

19 Enfin, cette particule est suivie du véritable nom propre du personnage, Ahecraruf, nom commun signifiant "précipice. Endroit difficile d'accès. Rocher élevé » (Dallet, 1982 : 304).

20 Ce nom à trois éléments est, en fait, constitué de deux parties : le nom du personnage proprement dit, Ahecraruf, et une partie surajoutée, que nous nous proposons d'appeler "définition ». Ce sont des mots que l'on rajoute au nom pour distinguer une personne. Il s'agit ici de ccix, qui peut indiquer soit la profession, soit le rang social, et de Illulen Umalu qui est un localisateur.

21 Nous en déduisons que la composition du nom est oxymorique : la partie « définition » annonce que l'on a affaire à une personne distinguée (sage, respectable), tandis que le nom propre suggère l'idée d'un parcours difficile, semé d'embûches. C'est sur ce contraste du personnage éponyme qu'est construite toute la pièce de théâtre. 


\section{Ssarid Bu Tlufa et Aqannun} prénom courant, Fațima, par troncation de la dernière syllabe. Par sa nature hypocoristique, et par sa combinaison avec la particule nna ("grande sœur » en berbère), il connote l'affection attachée au personnage Elise qu'Eugène appelle « chère Elise » dans l'œuvre originale. Ce nom permet aussi, par sa terminaison, de créer une résonance phonétique avec le nom de Wejțți qui entraîne une musicalité du titre.

\section{Sinistri et Sifuni}




\section{Eléments de comparaison}

De ce qui précède, nous pouvons retenir des points de ressemblance entre les deux types de production. Cela apparaît dans le choix des noms des personnages, dans leurs caractéristiques morphologiques et sémantiques ainsi que dans les registres linguistiques auxquels il est fait appel.

\section{Choix des noms}

Il y a beaucoup d'éléments communs entre des noms de personnages créés par Mohya et ceux des contes de la tradition orale. Citons les plus importants :

- les deux discours ne puisent pas leurs dénominations directement du registre courant des prénoms kabyles. Soit il est fait recours à une création toute nouvelle, soit, quand un nom courant est sollicité, il est complètement remanié ; ils ont des noms qui sont, morpho-syntaxiquement, très semblables. Par exemple dans la paire Beleejjuḍ/Wejțtuti, il est fait recours au même procédé de formation, la dérivation expressive, et, probablement aussi, à la même racine. La signification fondamentale de cette racine, qui renvoie à la faiblesse physique (évoquée plus haut), est utilisée dans le premier pour montrer qu'un être faible (Belcejjud) peut, grâce à son intelligence, vaincre un être beaucoup plus fort (l'ogresse). La même signification lexicale est utilisée, dans le second, pour rendre compte d'une certaine affectivité contenue dans la comptine française ;

- nous notons également une grande ressemblance dans certains noms composés. C'est le cas dans Eica Myizden/Ssazid Bu Tlufa : en dehors du genre (féminin pour le premier et masculin pour le second), les deux noms ont exactement la même structure, jusqu'aux types des composants : une partie dénominative (Eica/Ssacid) puisée dans les prénoms ordinaires empruntés au système onomastique arabe, et une partie définition ( $m m$ yizizden/bu tlufa), deux adjectifs formés par le même moule (le morphème $\mathrm{mm} /$ bu) ;

- dans ce dernier cas, mais aussi dans d'autres, on peut remarquer cette utilisation ironique, que certains auteurs appellent onomastique divergente ${ }^{11}$, qui consiste en la contradiction entre la signification lexicale du nom et la réalité sociale du personnage. Ainsi, Eica, "femme vive, active, vivante, dynamique », est une fille qui parait, en tout cas dans sa prime jeunesse, très naïve et sans vitalité, se contentant de jouer dans les cendres, alors que Ssacid, "heureux, fortuné, bienheureux (au paradis) " (Beaussier, 1958 : 474), a eu une fin de vie très malheureuse.

\section{Création et adaptation}

Les genres littéraires de la tradition orale en Kabylie sont essentiellement les contes, les fables, et surtout la poésie. Or, ce genre de production voyage souvent entre les contrées, et l'œuvre est, à chaque fois, enrichie, réaménagée, reformulée, adaptée aux conditions particulières de chaque localité, de telle sorte qu'en fin de compte, on ne puisse pas la distinguer des productions proprement autochtones. Ainsi, il existe certainement des contes qui sont d'origine kabyle, mais il y a aussi des contes empruntés à d'autres cultures et qui ont été adoptés par la société et adaptés à la 
culture locale. Par le seul nom du personnage principal, nous pouvons déduire que, par exemple, le conte dit Ssif Lyadin (" l'épée des deux mains ») est un emprunt à l'arabe. Ce nom désigne le personnage qui manie deux épées en même temps, une dans chaque main. Mais l'emprunt est si bien adapté que son origine est difficilement décelable. On retrouve la même situation dans l'œuvre de Mohya. Si ce n'était pas annoncé par l'auteur lui-même, peu nombreux seraient ceux qui décèleraient l'adaptation dans cette œuvre. Les noms et l'environnement sont si minutieusement recréés en fonction du contexte, que l'on croirait à des histoires typiquement kabyles.

Dans les deux types de production, il est fait appel aux ressources internes de la langue, mais les langues qui sont en contact avec le kabyle sont également sollicitées.

Ainsi, le conte a beaucoup recours aux emprunts arabes. Ceux-ci se font soit par l'utilisation de noms que le langage courant a lui-même adoptés (le cas de Eica), soit par l'utilisation de noms communs dont le sens est obscur dans le contexte kabyle, ce qui les rend parfaitement aptes à jouer le rôle de noms de personnages (Ssif Elyadin). Il peut s'agir aussi d'adaptation de personnages appartenant à la culture arabe (Ğehhha, Banwas...).

L'œuvre de Mohya est marquée par le recours à la langue française: il a créé des personnages en empruntant et en manipulant des mots français, comme évoqué plus haut.

Si, dans le théâtre moderne, il y a ce que l'on appelle la mise en scène, c'est-à-dire tout ce qui concerne la disposition des personnages, le choix et l'emplacement de tous les objets, des jeux de lumière, etc.; cela est remplacé, dans le conte et le théâtre audio, par la mise en condition de l'auditoire.

37 En raison de la nature audio de l'essentiel de son œuvre, Mohya recourt à différentes astuces pour captiver l'attention de son auditoire. Parmi les techniques utilisées pour mettre en condition son public, nous en avons relevé deux : les formules introductives qui jouent le même rôle que dans les contes et la manière de conter (insister sur un élément particulier, des répétitions bien ciblées, se moquer de certains personnages, le jeu sur les différentes tonalités de la voix,....).

38 Pour conclure, nous retenons que le conte de la tradition orale berbère a beaucoup inspiré Mohya, que ce soit dans sa poésie ou dans ses pièces de théâtre. C'est ce que nous avons décelé dans le choix des noms de ses personnages. Cette influence se lit dans le recours à des noms qui ne sont pas ordinaires, mais aussi à des créations spécifiques à chaque situation, comme dans le conte. Il en est de même des procédés de création des noms propres. Il est fait appel, dans certains cas, aux mêmes schèmes ou à la même racine lexicale.

Mais, à y regarder de plus près, on relève beaucoup de points de rupture dans ce qui semble être une continuité. Ce sont les mêmes points de ressemblance avec la littérature traditionnelle qui sont utilisés pour imprimer un saut qualitatif à la littérature amazighe et, ainsi, amorcer les changements nécessaires à son inscription parmi les productions dites universelles. 


\section{BIBLIOGRAPHIE}

ATOU Brahim, Toponymie et espace en Algérie, Alger, Institut National de Cartographie, 1998.

BEAUSSIER Marcelin, Dictionnaire pratique arabe-français, Alger, Carbonnel, 1958.

BEZZAZI Abdelkader, « Histoires de noms/noms d'histoires » in ABDELALI SABIA (dir.), Des noms et des noms, Publications de la faculté des lettres $\mathrm{N}^{\circ} 26$, Série : études et Séminaire’s $\mathrm{N}^{\circ} 9$, Université Mohamed Premier, Oudjda, 1999, pp. 77-93.

CAMILLI (de) David 1998, «Quelques noms de personnages dont le fort de Cesare Pavese » in BILly Pierre-Henri et CHAURAND Jacques (édition), Onomastique et Histoire. Onomastique littéraire, Actes du VIIIe colloque de la SFO (Aix-en-Provence, 26-29 octobre, 1994), Publication de l'Université de Provence, 1998, pp. 348-353.

CHAOUI, Mokhtar, « Le jeu patronymique chez Marcel Arland » in SABIA Abdelali, Des noms et des noms, Publications de la faculté des lettres $N^{\circ} 26$, Série : études et Séminaires $N^{\circ} 9,1999$, Université Mohamed Premier, Oudjda, pp. 29-46.

CHEMAKH Said, « D'une langue à l'autre ou l'œuvre de Mohya », in Tamazgha, 2004, disponible sur le site : [http://www.tamazgha.fr/D-une-langue-a-l-autre-ou-l-oeuvre-de-Mohya,1196.html]. CLERGET Joël, « L'essor du nom » in CLERGET Joël (dir.), Le nom et la nomination, source sens et pouvoir, Toulouse, Édition Eres, 1990.

DALLET J.M., Dictionnaire kabyle-français, Paris, SELAF, 1982.

GARY-PRIEUR Marie-Noëlle, L'individu pluriel : Les noms propres et le nombre, Paris, CNRS, 2001.

MOHEND-OU-YAHIA, « interview », in Tafsut, 1985, reproduite depuis 2004 dans la revue en ligne tamazgha, disponible sur le site : [http://www.tamazgha.fr/Entretien-avec-Mohya-dans-Tafsut, 960.html].

TDJET Mustapha, La Patronymie dans les dairas de Timezrit, Sidi-Aich et Chemini. Etude morphologique et sémantique, Thèse de Doctorat, soutenue à l'université Mouloud Mammeri - Tizi Ouzou, en janvier 2013. Disponible à la bibliothèque de l'université.

VAXELAIRE Jean-Louis, Les Noms propres : une analyse lexicologique et historique, Paris, Honoré Champion, 2005.

\section{NOTES}

1. MOHYA Abdallah dit Muhend u Yehya (1950-2004) est un écrivain-traducteur algérien de langue berbère. Son œuvre se compose essentiellement de poèmes, de contes, de nouvelles et de traductions en berbère de pièces théâtrales du patrimoine mondial.

2. Ce terme est pris dans un sens nettement péjoratif que le poète assume en précisant sa pensée à la fin du même paragraphe : «Et je dis ceci en tout état de cause, dans la mesure où les faiblesses et les maladresses qu'on ne manque pas d'y relever sont elles-mêmes significatives du niveau culturel des gens de chez nous » (Mohya, 1985).

3. Mqidec est utilisé tel quel comme patronyme ; il n'a subi aucune modification à l'exception de la transcription française de sa phonie : Mekideche. 
4. Le patronyme de Lounğa n'est pas retrouvé tel quel, mais « habillé du schème arabe de filiation au masculin : Loundji » (Tidjet, $2013: 220$ ).

5. Nous disons qu'«ils paraissent ordinaires", mais, en fait, ils ne le sont pas. En effet, contrairement aux prénoms kabyles qui sont toujours formés sur des bases lexicales attestées en langue (en dehors des prénoms empruntés à l'arabe), ce nom n'appartient, nous semble-t-il, à aucune famille lexicale. De plus, malgré les connotations positives qu'il véhicule, il n'est jamais attribué dans la vie réelle.

6. Selon J. M. Dallet, ce mot n'est pas attesté comme nom commun chez les At Mengellat, mais plutôt comme nom de lieu : Tamejjut « nom d'un village des At Mangellat », amejjud « un homme de ce village » (Dallet, 1982 : 493). La gale se dit ajeğğị (Dallet, 1982 : 361).

7. En arabe, ceyx est polysémique : « âgé, vieux, vieillard, vieil homme. Ancien (par rapport à l'âge et à la dignité). Cheikh, chef de fraction, de tribu. Directeur d'un collège, d'une zaouïa. Maître, professeur. Maire. Beau père de la femme » (Beaussier, 1958 : 553) ; et selon Atoui (1998) : "Qui est expérimenté, d'où homme qui exerce une autorité morale ou politique. Administrativement : fonctionnaire, notable, responsable du douar, de la tribu» (242).

8. Par extension, il réfère aujourd'hui à "maître ». Pour éviter tout amalgame, on précise en général la profession, ainsi ccix l-lacul « maître d'école », et ccix tout court ou ccix $n$ taddart pour l'imam du village.

9. Le texte intégral de la comptine est disponible sur le site "Comptines et poésies»: materalbum.free.fr/poesies $3 . h t m$

10. Ce morphème se combine avec un nom pour former un adverbe : ayyul «âne » et yyuli «à la manière d'un âne ", « bêtement ».

11. Selon Chaoui M. (1999), "Cette approche onomastique consiste en ce que les patronymes choisis traduisent exactement le contraire de ce que leur dénotation signifie. (...) L'onomastique, dans ces cas-là, devient une onomastique ludique, mais qui n'est pas dénuée de signification ou de symbole. L'ironie qu'elle recèle n'est autre que l'ironie du sort... (Chaoui, 1999 : 41-43).

\section{RÉSUMÉS}

A travers cette étude des noms propres, nous tentons de montrer que l'œuvre de MOHYA ${ }^{1} \mathrm{~A}$. est, par certains aspects, influencée par la tradition orale berbère. Il recourt, par exemple, aux mêmes procédés de formation des noms : la dérivation expressive et la composition. Par la dérivation, il crée de nouvelles unités n'appartenant pas au registre commun, et par la composition, il transforme les noms ordinaires en les combinant avec de nouveaux composants ; le nom ainsi remodelé entretient une relation motivée avec le personnage dont il programme la lecture, la personnalité et le destin narratif. Cependant, nous analysons également, mais de façon succincte, les autres aspects novateurs de l'œuvre de cet écrivain : les codes génériques utilisés et les thèmes traités.

Through the present study of proper nouns, we attempted to show that Mohya's work is, in some of its aspects, influenced by the Berber oral tradition. He used, for instance, the same nouns forming processes: expressive derivation and composition by derivation, he creates new units which do not belong to the common register, and by composition, he transformed ordinary nouns by combining them with new components; the such remodeled noun undertakes a motivated relationship with the character that he plans reading, personality and narrative 
destiny. However, we also analyzed briefly, the other innovating aspects of this author's works: the used eneric codes used and topics dealt with.

INDEX

Mots-clés : onomastique amazighe, linguistique amazighe, conte berbère, formation des noms, Mohya Abdallah

Keywords : Amazigh onomastics, Amazigh Linguistics, Berber tale, nouns forming, Mohya Abdallah

\section{AUTEUR}

MUSTAPHA TIDJET

Université A. Mira Bejaia - LAILEMM 